\title{
The potential of a thick present through undefined causality and non-locality
}

\author{
Alessandro Capurso \\ Feb. 8, 2022 - Rome, Italy
}

\begin{abstract}
This paper elaborates on the interpretation of time and entanglement, offering insights into the possible ontological nature of information in the emergence of spacetime, towards a quantum description of gravity. We first investigate different perspectives on time and identify in the idea of a "thick present" the only element of reality needed to describe evolution, differences, and relations. The thick present is connected to a spacetime information "sampling rate" and is intended as a time symmetric potential bounded between a causal past of irreversible events and a still open future. From this potential, spacetime emerges in each instant as a spacelike foliation (in a description based on imaginary paths). In the second part, we analyze undefined causal orders to understand how their potential could persist along the thick present instants. Thanks to a C-NOT logic and the concept of imaginary time, we derive a description of entanglement as the potential of a logically consistent open choice among imaginary paths. We then conceptually map the imaginary paths identified in the entanglement of the undefined orders to Closed Time-like Curves (CTC) in the thick present. Considering a universe described through information, CTC are interpreted as "memory loops", elementary structures for the persistence of the information potential related to the entanglement in both time and space, manifested as undefined causality and non-locality in the emerging foliation. We conclude suggesting a possible extension of the introduced concepts in a holographic perspective.
\end{abstract}

Keywords: time; presentism; causality; free will; entanglement; CTC; information; quantum gravity;

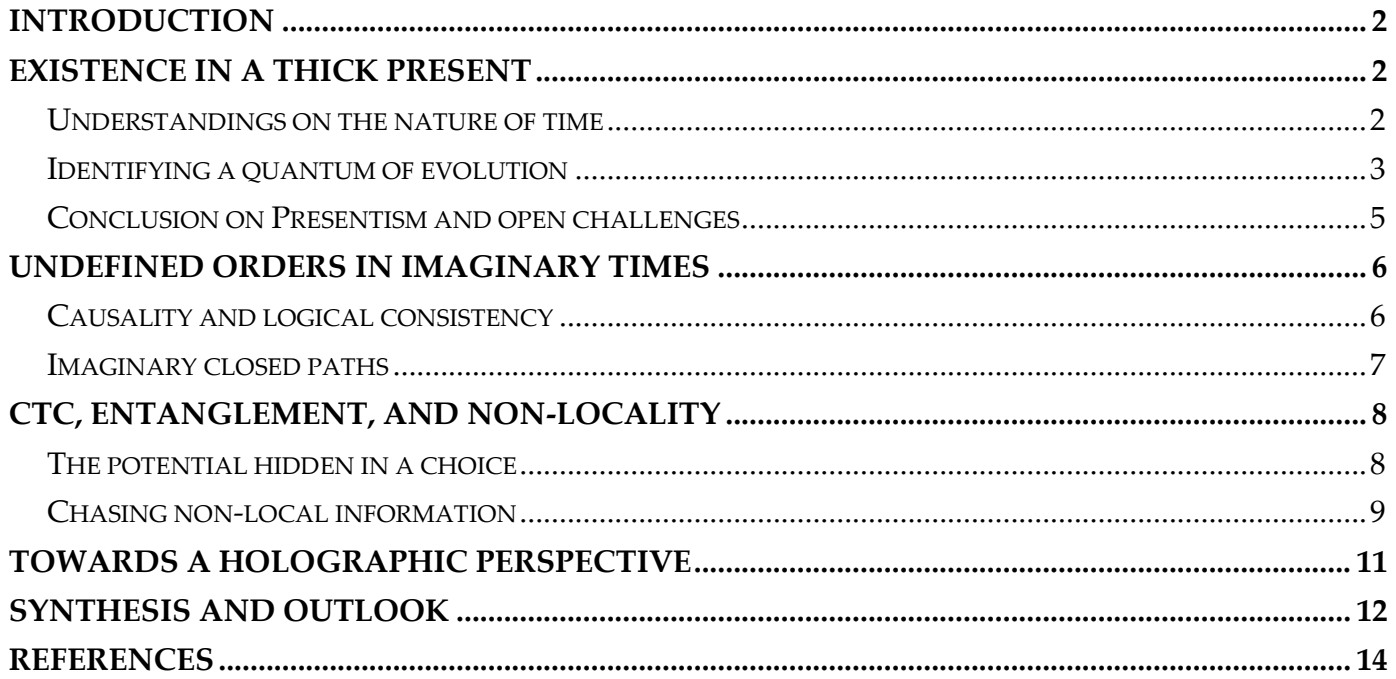

\footnotetext{
\# ale.capurso@gmail.com - orcid.org/0000-0003-3277-3588
} 


\section{Introduction}

The nature of Time is often at the root of the debate in physics and possibly sits at the core of General Relativity (GR) and Quantum Mechanics (QM) incompatibility. In recent years, the search for a theory of Quantum Gravity (QG), able to include the success of both GR and QM, revived the study of time as a key ingredient for the understanding of a quantum description of spacetime.

After an investigation on multiple perspectives on the subject, this paper suggests the interpretation of time through the concept of a time symmetric "thick present". Within each thick present instant, intended as the only element of reality along an emerging axis of thermodynamic and causal time, a quantum information potential $T_{k}$ is considered, from which spacetime emerges in a sequence of spacelike foliations.

Beside time, the concept of entanglement has puzzled the physics community for decades, stimulating the discussion around causality and locality.

In an evolution occurring in discrete instants, we investigate how indefinite causal orders (as entanglement in time) could be described.

We first consider undefined causality through a parallel with a C-NOT quantum gateway. Following a path integral approach, we describe the information in the undefined order through entangled imaginary paths in the C-NOT circuit, which develops as superposed imaginary times in each spacelike foliation. The superposition of imaginary times in a time symmetric potential is finally interpreted as a closed path (CTC) in the thick present.

In the context of a Quantum Information Science (QIS) description of spacetime, CTC@ $T_{k}$ are interpreted as logically consistent "memory-loops", in which the information potential of an "open choice" (temporal order or spatial position) could persist along the succession of the present instants. We conclude suggesting a connection between entanglement (EPR), non-locality $(E R)$ and CTC in the thick present through a holographic perspective.

\section{Existence in a thick present}

\section{Understandings on the nature of time}

The nature of time has often led to confusion, as several different meanings have been associated to the word "time", both from neuroscience and physics, as illustrated by Buonomano and Rovelli in Ref. [1]. The debate is often reduced to two opposite and extreme interpretations. The first, called Eternalism, considers an ever-existing time, real in both the past and the future, and of which we perceive only limited cones of information. The opposite perspective, known as Presentism, pictures time as a vanishing present compressed between past and future instants. The focus is on the difference between the past, already happened and irreversible, and the future, still undefined and open.

Neuroscience considers a forward and causal progression of time: a fixed past and an undefined future, both not-existing as only the subjective experience of "here and now" is given as "real". Our neurons equalize different asynchronous stimuli and derive a coherent picture of the surrounding space in each "perceived instant", remembering the past events. Our perception is not always physically accurate but to our brain looks coherent and this is enough for a perception of a "flowing time": a past connected to the future through what is happening in the present. It could be considered a "local presentism", with no interest for the interpretation of time at quantum or cosmological scales, but the wish to reconcile the emerging human perception with more fundamental phenomena.

Physics, from thermodynamics, considers the arrow of time as "emerging on average" or macroscopically (but not fundamental). Newtonian mechanics is compatible with "global presentism", as a "perceived now" common to all entities, but the relativity of time in GR seems to preclude this possibility.

From GR, time is described as intertwined with space (in a relativistic spacetime), but space and time are anyway different concepts, related to a clearly identifiable "space separation" between events (no causal influence) or "time separation" (possible causal influence). 
Even if GR is our best theory on spacetime, we should consider its insights with caution: being a classical theory, GR should at least be incomplete. QG approaches are investigating "time-less" scenarios and further studies may show that a relativistic description of time is emergent from more fundamental principles.

Finally, Planck units strongly suggest a discreteness of spacetime, highlighting the existence of absolute references derived from fundamental constants in our Universe.

From a QM and a logical perspective, in the context of the Free Will theorem (introduced by Conway in [2]), we should assume that the future is undetermined and pose a serious threat on the idea of a classical and ever-existing time in a "block-universe". As Freeman Dyson wrote in [3], the only role of a local observer seems to be the distinction between a classical past and a probabilistic future. The relation between the observation of a variable at a given instant (identifying an event) and the derived difference between past and future has also been elaborated by Smolin in [4] as a "dynamic of difference".

From a QIS perspective, recent "gargantuan simulations" (reported in [5]) showed that time seems irreversible at the most fundamental level, beyond thermodynamic reasons. It has been shown that even a simple 3-bodies system "would require an accuracy of smaller than the Planck length in order to produce a time-reversible solution": what had been already can causally influence the present but cannot be changed and, beyond fundamental physical limits, cannot even be known with certainty.

The irreversibility of events might seem in contradiction with a symmetric description of time, even if physical laws in a classical framework are time symmetric and there are several QM symmetric approaches too. The idea of an emerging reality connected to the superposition of both a forward and a backward propagating wave goes back to the Fifties, introduced by Watanabe in Ref. [6] as Two-State-Vector Formalism (TSVF).

In recent years, in the context of a time symmetric approach, the concept of irreversibility has been more clearly connected (in Ref. [7] and [8]) to the idea of irretrodictability from a logically consistent Bayesian perspective. The emergence of a causal arrow of time from logical consistency has also been investigated in Ref. [9].

Additional insights on a time symmetric description with elements of Energetic Causal Sets has been developed by Cohen et al. in [10] and [11], further smoothing the tension between a causal and irreversible perspective irremediably opposed to time symmetry.

\section{Identifying a quantum of evolution}

If the future is open and yet to come, the past is irreversible and might not be even known beyond certain limits and time shows a level of symmetry in its evolution, we could consider a time symmetric thick present as the elementary quantum in the passage of time. A thick present could be defined as the information $T_{k}$ related to a thick spacelike foliation, bounded by $-T$ and $+T$ and derived from a time-symmetric superposition of perspectives (from a near past and a near future) on the emerging spacetime.

Within a thick present we could consider both the quantum information potential (in a time symmetric description) as well as the information of the last events (intended as causal points at the past bound of the thick present, from which the present emerges and the future opens), efficiently discarding (for Occam's sake) all the information that is not "currently needed" to describe the evolution of the Universe.

A thick present is the actual realization of a discreteness of time and, from an ontological perspective, shall be intended as the only element of reality in a logically consistent, causally and thermodynamically oriented emerging axis of an extended classical time.

It is worth to note that, as highlighted by Tallant and Ingram in. [12], a well-defined philosophical framework of Presentism is missing, as several (and sometimes contradicting) descriptions are proposed in the literature. Among the many, in this contribution we will consider the definition stating that "Only the present time exists (No non-present time exists)". Following a philosophical perspective grounded on the physics of irreversible events, open future and indeterminate present, a Presentism interpretation of time has also been recently promoted by Mariani and Torrengo in Ref. [13]. 
In the search for a quantum description of space and time, a thick present has been considered by Gisin in Ref. [14] (via an intuitionist mathematical language), and by Smolin in Ref. [15] (from an ontological perspective in QM).

The concept of an everchanging "becoming" between a fixed past and a probabilistic future has been investigated also by Schlatter. Starting from a principle of synchronization, the gravitational potential is connected in [16] to a foliation of spacetime in spacelike surfaces and, sequencing the "flow of reality" in time intervals, the established relations between energy, entropy and geometry are recovered. In Ref. [17] and [18], events are interpreted through synched "light clocks" (introduced in Ref. [19]) in an emerging thermal time, and evolution is intended through a "realm of probability amplitudes" (with a symmetric time structure) and an emerging "empirical spacetime" (as events break the unitary symmetry).

A thick present could be related in QIS to a discrete elaboration of the global information potential in the spacelike foliation. There are several theories that consider evolution in discrete steps. To mention a few, Finite State Classical Mechanics (described by Margolus in Ref. [20]) is based on Lattice Dynamics, where evolution rules are often referred to as "cellular automata" models. Signal-State Quantum Mechanics, developed in a theory of Quantized Detector Networks (presented in [21]), is a realization of the Heisenberg's "instrumentalist approach" to quantum physics.

Following the insights from QIS and the famous "it from bit" (that considers spacetime and $\mathrm{QM}$ as emerging from a quantum information processing), Operational Probabilistic Theories (OPT), developed by Hardy, D'Ariano et al. (see Ref. [22], [23] and [24]), describe the evolution of quantum systems as logical-physical circuits that could be foliated in hyper-surfaces elaborated in atomic steps. OPT have been considered in a time symmetric perspective (in Ref. [25] and [26]) and in terms of a difference between known and unknown, rather than an emerging past and future (as in Ref. [27]).

Even if the idea of a thick present has not been explicitly considered yet in the context of an OPT description of spacetime, we could identify in the atomic processing of OPT the realization of an atomic thick present in the evolution of the system, and then map the space foliation emerging in each processing cycle to an equivalent circuit-foliation.

To describe a time symmetric thick present in the context of a discrete evolution of the information (phased on atomic cycles), we should consider a minimum time interval $T$ like a $\pi$ rotation, and $2 T$ for a full cycle in a time symmetric description. We could consider these discrete $2 T$ steps, from $(2 k-1) T$ to $(2 k+1) T$, as the atomic elaboration cycle of the present information $T_{k}$ from which spacetime emerges as spacelike foliation at $2 k T$.

It is worth to clarify that the concept of "spacetime from information" is not promoting the idea that "we live in a simulation", which is an unneeded speculation. Moreover, the "present" is not intended as a "global perceived now". The passage of time for local systems follows relativity and time intervals measured by local quantum clocks through events vary according to GR, as there is no absolute perspective for any local observer within the emerging spacetime, but only relative ones. The "perceived now" of quantum systems (from particles to complex clocks), shall be intended as a "proper evolution cycle" of the system, measured on to the past cycles and of greater duration compared to the thick present extension, as the spatially non-local "here" that spreads in the wave-function.

The thick present $T_{k}$ represents the potential of the $k^{\text {th }}$ space of events and possibilities, as a thick spacelike foliation bounded within $(2 k-1) T$ and $(2 k+1) T$. In a QIS picture, its duration $2 T$ should be intended as a spacetime information "sampling rate". The idea of a maximum rate of change connected to the inverse of the Planck time has also been elaborated in [28], where it has been proven to be compatible with Relativity. In this context, the thick present should be interpreted as a global reference for time intervals to allow relative confrontation of quantum clocks with respect to one another in a discrete passage of time. Relational time (defined in [29] as the "counting of happenings") still needs an "elementary event" to allow independent clocks to compare their "counts" in a coherent and consistent way, as absolute references are always required for uniformity in comparisons. In this sense, the present information processing cycle represents a quantum of elementary action (the fastest event to evaluate differences) available as a reference on which the Universe fabric evolves. 
Observers, events or potential ones, all could coexist in a thick present able to account for a superposition of perspectives on the information potential from a near past and a near future, resulting atomic and time symmetric within its thickness, and assuring consistency between "what it was" (causally happened) and "what it could be" in the current cycle.

The information persists and evolves as a potential in case no specific events occurred in the present elaboration cycle, while events of decoherence shall be seen as irreversible, also in line with the QIS and causal set perspective.

From the irreversibility of events, a thermodynamically oriented arrow of time in line with causality can also be considered as emerging in the succession of the thick present instants.

\section{Conclusion on Presentism and open challenges}

A Presentism perspective on time has ancient origins, it is rooted in several western and eastern philosophies and it is coherent with the latest interpretations in neuroscience. Relativity, allowing a description of reality focused on local observers, has then thought us that every description of reality could and must be relative, dissolving an absolute passage of time in a relativistic spacetime fabric. The multiple relative perspectives on the same information in terms of events have mined the concepts of before and after, leaving to an absolute speed of propagation of the causal information the role to preclude paradoxes. Eventually, in a relativistic description, time appears in an extended classical reality of past events and a deterministic future, seemingly staving off a Presentism perspective from all physical interpretations.

Recently, in the context of relativistic time intervals but beyond the limits of a classical description, part of the physics' community has tried to reconcile the interpretation of time with the ancient philosophical understandings, realizing that in the indeterminacy of the quantum information lives the potential of the present instant, causally dependent on the set of past irreversible events and door to an open future.

In the first part of this contribution, we have presented the main insights towards a description of time coherent with relativity and QM and with a Presentism perspective. In the context of QIS, we have proposed an interpretation of the present as the information in the $k^{\text {th }}$ evolution cycle of the space of events and possibilities, emerging as a spacelike foliation, bounded between $(2 k-1) T$ and $(2 k+1) T$ in a time symmetric description.

The temporal extension of the thick present, actual realization of a discreteness of time, has been interpreted as a spacetime information "sampling rate". The atomic elaboration of the information potential in each instant has been proposed as a quantum of elementary action, absolute reference needed among independent observers or clocks to consistently define and compare any relativistic perspective on the information evolution.

In the framework of a thick present, Classical is then what we remember or causally predict, it is what has already happened or will happen if there were no quantum features. Complex observers may encode in the complexity of their internal dynamics the information of the past events and derive a corresponding thermodynamic and causal orientation of a classical time.

Classical reality emerges from the information encoded in the memory of complex observers but should not be intended as "currently real": in an ontological sense, it does not exist. From the proposed perspective, the Universe exists in the thick present only.

Figure 1 graphically illustrates the introduced proposal.

In the given interpretation, the information $\boldsymbol{T}_{k}$ plays a crucial role in the emergence of spacetime and a better description of how this potential is encoded in the thickness of the present is needed. Moreover, recent experiments have shown that undefined causality (entanglement in time order) is possible.

In the following chapter we will investigate the relation between causality and logical consistency and propose how the information potential of entanglement in time orders could be intended and persist along the succession of the thick present instants.

In the final part of this paper, we will conjecture a possible relation of the information potential with undefined locality and entanglement in space in a holographic perspective. 


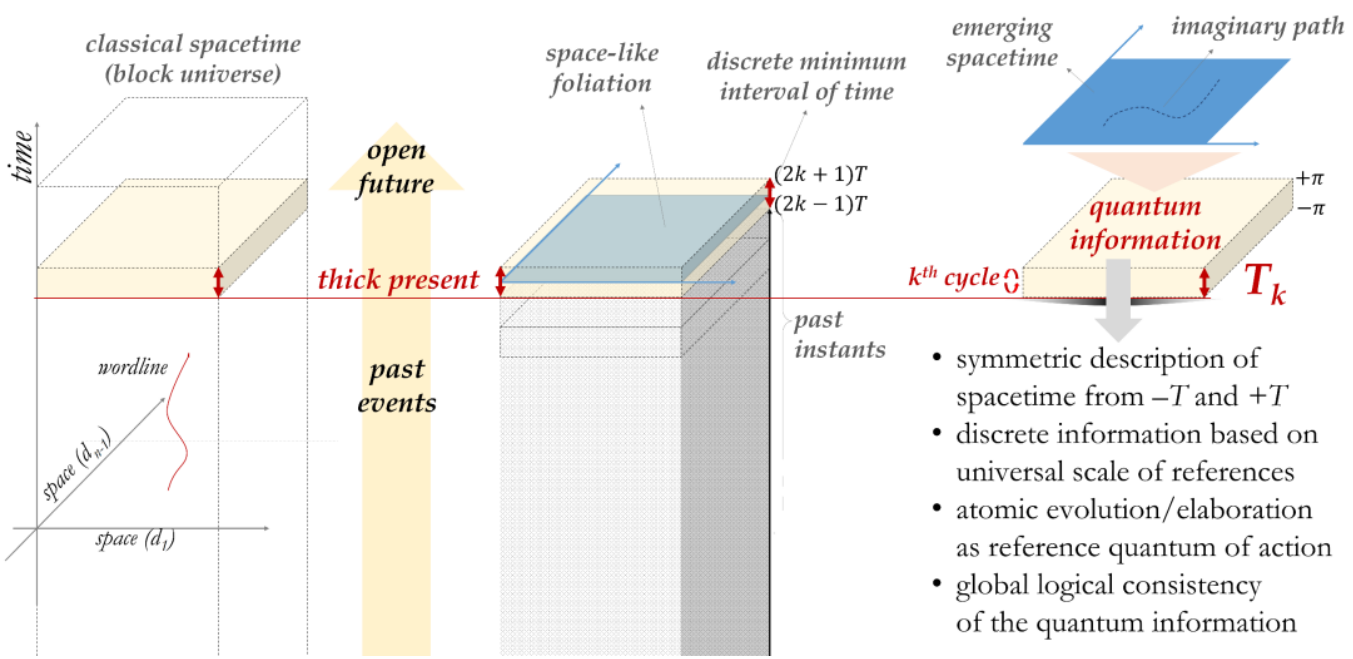

Figure 1. Identification of the thick present as the current thick spacelike foliation and corresponding $k^{\text {th }}$ elaboration cycle of the quantum information $\boldsymbol{T}_{k}$ from which spacetime is considered emerging.

\section{Undefined orders in imaginary times}

\section{Causality and logical consistency}

Recent investigations in the physics of time highlighted the possible existence of Undefined Causal Orders (UCO) and derived the equivalent Bell's inequalities in terms of temporal orders (as illustrated by Brukner et al. in [30]). Entanglement of temporal orders and experimental verification of UCO were considered as well in [31], [32] and [33].

In an evolution described as occurring in thick present instants (given as the only element of reality in time), it is worth understanding how UCO could occur and how their potential, in the entanglement of temporal orders, could be intended.

The authors of [32] identify a "quantum SWITCH" circuit able to selectively choose the route of a particle, so that Alice $(A)$ is encountered along the path before Bob $(B)$ or vice-versa, depending on a controller qubit $C$.

The quantum SWITCH circuit could be described, from a logical perspective, as a device able investigate the alternative scenarios " $A$ happens before $B$ " or " $B$ happens before $A$ ", equivalent to " $A(B)$ is first in time and $B(A)$ is not first", through a controller qubit which is in a superposition of states.

In circuit logic, the same behavior could be described through a XOR function $A \oplus B$, given that " $A(B)$ is true" when " $A(B)$ is met first in the path". Given $A$ and $B$ as any pair of points along the path of a particle entering the circuit, the XOR gate superposes the 2 statements " $A$ is first, and $B$ is not first" and " $B$ is first, and $A$ is not first". The resulting information of the XOR function is true if one and only one of the two assumptions is true, excluding the under-determined scenario (both false, as if there were no "first") or, on the other hand, an over-determined solution (both true, as if both were "first").

The XOR gate assures logical consistency of the global information in the context of an "open choice", limiting the possible outcomes to the only ones which imply a difference, and excluding the logical paradoxes of over/under-determined solutions.

In quantum logic, the XOR function could be described as a Controlled-NOT (C-NOT) quantum gate, which operates on a quantum register consisting of 2 qubits $C$ and $S$ (Controller $C$ and Target $S$ ) and flips the qubit $S$ if and only if $|C\rangle=|1\rangle$.

The C-NOT gate correlates the information potential of the controller qubit $C$ with the information potential of the Target $S$ (the particle entering the circuit), and it is a common system used to create entangled pairs. For instance, an experiment in which a particle changes one of its quantum properties depending on the direction of travel in the circuit would create an entanglement between the observable in the particle and in the controller qubit, as if the potential information of the path of the particle were locally "stored" in $C$. 
A similar description of the quantum SWITCH is given in [34] as a quantum time flip device. The proposal is elaborated in the context of OPT and unitary operations and it has been implemented through entangled photons.

It is crucial to highlight that, given the quantum superposition of the 2 circuit paths along the opposite directions, the "choice" could not happen immediately, at the time of the first $C$-S interaction. We need a fixed event to have a definite and irreversible outcome of the choice and, as long as the particle or the controller are not observed (and contextually defined), both results are possible: the outcome of the choice is kept as a quantum information potential in the relation between $C$ and $S$, as illustrated in Figure 2.

\section{Imaginary closed paths}

To picture an undefined order within a thick present and the corresponding spacelike foliation, the path of the particle inside the circuit should be described as a function of the propagation velocity $v$ and of an imaginary time of motion needed to traverse the circuit (as a dimension of possible freedom), in a resulting imaginary path $i v \tau_{|C\rangle}$ along the circuit. We should consider the point $C$ as the position of the controller qubit closing the logical circuit in the spacelike foliation, while $A(B)$ could be defined as the imaginary point reached at the imaginary time $i \tau_{|C\rangle}$ when propagating in the anticlockwise $\left(i \tau_{|1\rangle}\right)$ or clockwise $\left(i \tau_{|0\rangle}\right)$ direction along the circuit.

The point of entrance of the particle in the circuit (as the instant of entanglement with the controller $C$ ) becomes the point in the past of the particle and of the controller in which an information potential related to a consistent and still "open choice" was established.

The concept of an imaginary time has been popularized by Hawking in Ref. [35] and could be interpreted as a Wick rotation (able to offer a Euclidean description of the Minkowski metric) which is common in the Path Integral (PI) formulation of QM.

In each instant, a spacelike foliation could be described from the information in the causal set of events $F$ ("fixed past choices" already defined at the past boundary of the present), in the points of quantum interactions generating potential $O$ (as new "open choices"), and through an imaginary time of motion (as imaginary paths) emerging from them.

Considering an imaginary time of motion $\left(i t_{F, O}\right)$ needed at the speed of light from any quanta of space, the imaginary paths traced along $i c t_{F, O}$ (or ict for short) can define and trace an imaginary Minkowski space within the thick present.

A possible description of spacetime as spacelike foliations in a PI context could rely on the definition of a new Hilbert space $\mathcal{H}$ built upon the tensor product of copies of the conventional Hilbert space $\mathfrak{h}$, one for each elaboration cycle $\left(\mathcal{H}:=\bigotimes_{k} \mathfrak{H}_{k}\right)$, and then on the application of the related unitary time translation operator along the successive slices, as elaborated in Ref. [36]. The description of a relativistic particle in a PI formalism has been discussed in [37], where the Feynman propagator has been connected to the imaginary action in the spacelike paths, to be accounted in the sum of all possible trajectories together with the orthochronos paths (at the speed of light).

Even if far from a proper derivation in a PI formalism, we could still consider the imaginary path $i v \tau_{|C\rangle}$ (in entanglement with the controller $C$ ) defined after the time of traversal of the C-NOT circuit as the representation of the quantum information connected to the open choice established in the undefined causality.

Modelling the entanglement in the undefined orders through a C-NOT quantum logic and superposed imaginary paths developing in an imaginary time of motion in opposite directions along the circuit allows a novel interpretation of the phenomena.

From the perspective of an imaginary time developing in the time symmetric thick present, the superposition of the paths $\left(i v \tau_{|1\rangle} \oplus i v \tau_{|0\rangle}\right)$ could be indented as the logically consistent superposition of a forward and a backward evolving wave persisting in the circuit, as well as an imaginary Closed Time-like Curve (CTC) connecting the point $A, B$ and $C$ (Figure 3). The suggested relation between CTC in the thick present and entanglement is open to several interpretations and needs further elaborations, left to the coming chapters. 

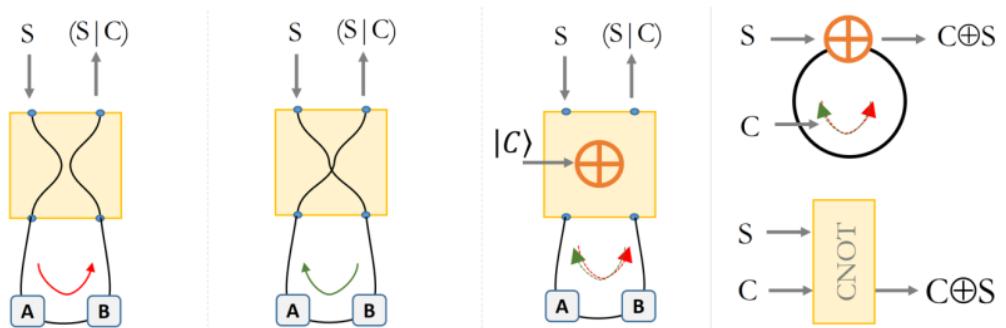

\begin{tabular}{|ccc|}
\hline $\mathbf{C}$ & $\mathbf{S}$ & $\mathbf{C} \oplus \mathbf{S}$ \\
\hline$|0\rangle$ & $|0\rangle$ & $|0\rangle$ \\
\hline$|0\rangle$ & $|1\rangle$ & $|1\rangle$ \\
\hline$|1\rangle$ & $|0\rangle$ & $|1\rangle$ \\
\hline$|1\rangle$ & $|1\rangle$ & $|0\rangle$ \\
\hline
\end{tabular}

$$
\begin{gathered}
|C\rangle=|1\rangle \text { Order: } \mathrm{A}, \mathrm{B} \quad|C\rangle=|0\rangle \text { Order: } \mathrm{B}, \mathrm{A} \quad \mathrm{UCO} \text { entangled } \\
P(A)=1 ; P(B)=0 \quad P(A)=0 ; P(B)=1 \quad \text { with }|C\rangle \\
P(X)=P(X \text { is met first })
\end{gathered}
$$

Figure 2. Controlled quantum SWITCH reproducing an UCO described as a XOR function, implemented as a C-NOT quantum gate. The entanglement of the Controller qubit $C$ with the Target $S$ at the point $\oplus$ allows the superposition of paths in which " $A(B)$ is met first and $B(A)$ is not", and consequently the UCO. Entanglement in the C-NOT gate could be seen as the information potential of a choice instantiated in the instant of the interaction and of which the answer is undetermined.

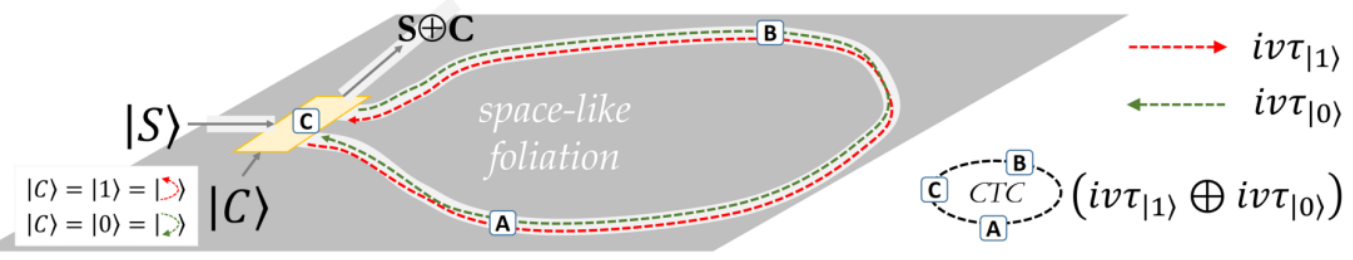

Figure 3. Superposition of the imaginary paths of the particle $\left(i v \tau_{|1\rangle} \oplus i v \tau_{|0\rangle}\right)$ in a spacelike foliation at a given instant after the time of traversal $\Delta \tau$. The result could be described as the superposition of a forward and backward evolving wave in the imaginary time of motion $\left(i v \tau_{|C\rangle}\right)$ and a CTC closed between $C$ and any two points $A$ and $B$ on the circuit.

\section{CTC, entanglement, and non-locality}

\section{The potential hidden in a choice}

CTC have been studied since the early days of GR as possible solutions to Einstein's field equations (Gödel in [38]) and their existence raised concern and suspect (as in [39]). Following the interest for a quantum description of GR, the nature of time gained momentum in the physics' research of the recent years, together with the CTC puzzles. It has been shown that CTC are incompatible with a causal and thermodynamic progression of time and that events cannot happen along their path (further elaborated in [40], [41] and [42]). Nevertheless, if time (as a progression related to causal events) cannot be considered on a CTC, the idea of a CTC developing along superposed imaginary times (within the spacelike foliation) could represent the information potential of the system in the thick present. In the proposed interpretation, the superposed possibilities in the entanglement are considered as the information of a logically consistent "open choice", discarding under/over-determined solutions in the XOR associated to the choice. This information persists in the CTC@T $k$ as a potential of superposed values of the outcome of the choice and eventually a quantum state with no identification of any event. In this sense, a CTC developing in the imaginary time represents an undefined causality in the thick present and an entanglement in the time order of the possible events in the closed path.

Without posing restrictions to the thickness $2 \mathrm{~T}$ of the present, we should consider a very fast "sampling rate" of the information (from which the difference between "something happened" or "nothing happened" is evaluated), and then, given a time of traversal $\Delta \tau>>2 T$, we should investigate what is happening while the particle is traversing the circuit. 
The description of the space foliation emerging in the thick present through an imaginary time of motion and an information potential related to "open choices" between imaginary points on a CTC may promote an idea of non-locality in the emerging imaginary space through its "thickness in time".

The particle, while traversing the circuit, should be considered as propagating in both arms (being in both a forward and backward propagating wave), and it is potentially on all the points already traversed along the curve in each thick instant. The path is then closed through a CTC developing in the thickness of the present (orthogonal to the space distance axis ict) representing a non-local correlations between the entangled possible imaginary locations of the particle within the imaginary space (as in Figure 4).

The relation between causal non-separability and cyclicity of the causal structure has also been highlighted in Ref. [43] and a connection between UCO, non-locality and closed curves in the context of logic games has been investigated in Ref. [44].

Still, a description of the information potential as CTC developing along an imaginary time in a symmetric thick present seems novel among the interpretations of entanglement.

In a QIS perspective, we can consider these CTC@T $T_{k}$ as spacetime "memory-loops", able to "encode" the quantum information potential of a logically consistent choice in spacetime, of which the outcome is open at the most fundamental level.

\section{Chasing non-local information}

Non-local information shall not allow faster than light communications (intended as a transmission of a message with a non-random information). Moreover, considering a finite speed for the causal propagation of information and that two experimenters (separated in a spacelike way) can make choices of measurement independently of each other, even in the context of entanglement, the Free Will theorem already introduced concludes that the result of any quantum observation cannot be fully determined by anything previous to the experiment.

Given the Free Will theorem and considering Bell and Kochen-Specker theorems (reported in Ref. [45], [46]), we shall remember as well that QM interpretations based on non-locality must be contextual: the value of a variable is determined considering the interaction with the local system involved in the measurement.

We should consider that it is in the fundamental randomness of the quantum observation that a "faster than light communication" finds its impossibility (even in the case of non-local correlations), and that a logical consistent causality is preserved.

The status that is "instantaneously updated" at the distant location $C$ by a measurement on $S$ would be coherent with the state of $S$ but, to a local observer at $C$, would appear as determined by a random process and so unable to carry meaningful information. Still, from a global and logically consistent perspective in $T_{k}$, the identification of a choice represents a quantum information potential persisting in the superposition of the outcomes.

In the thick present potential $\boldsymbol{T}_{k}$, entanglement in time order (as UCO) as well as among particles in different spatial locations (EPR pairs) could be equivalent to a CTC, described as a logically consistent "memory-loop" able to store the information potential of an open choice (offered in the entanglement) that precludes under or over-determined solutions. Loops are, actually, the most basic circuits for information storage and CTC@T $k$ could be considered as a "spacetime quantum memory" to encode the potential in $\boldsymbol{T}_{\boldsymbol{k}}$.

The proposed interpretation of an imaginary space in which non-locality is assured thanks to a thickness in time could offer insights into the "measurement problem" or the "collapse of the wave function". In the thick instants in which the choice's outcome is still open, the information potential propagates as a CTC orthogonal to the imaginary space, while selects one branch of the CTC and defines a causal path in the instant of observation. In the case of an EPR pair sent to Alice and Bob, the correlated information propagates superposed in the CTC. When Alice freely choses to observe her particle, she contextually defines a measurement event, which defines a determined orientation in the former CTC that opens in a causal path, ensuring logical consistency with Bob's measure (Figure 5). 


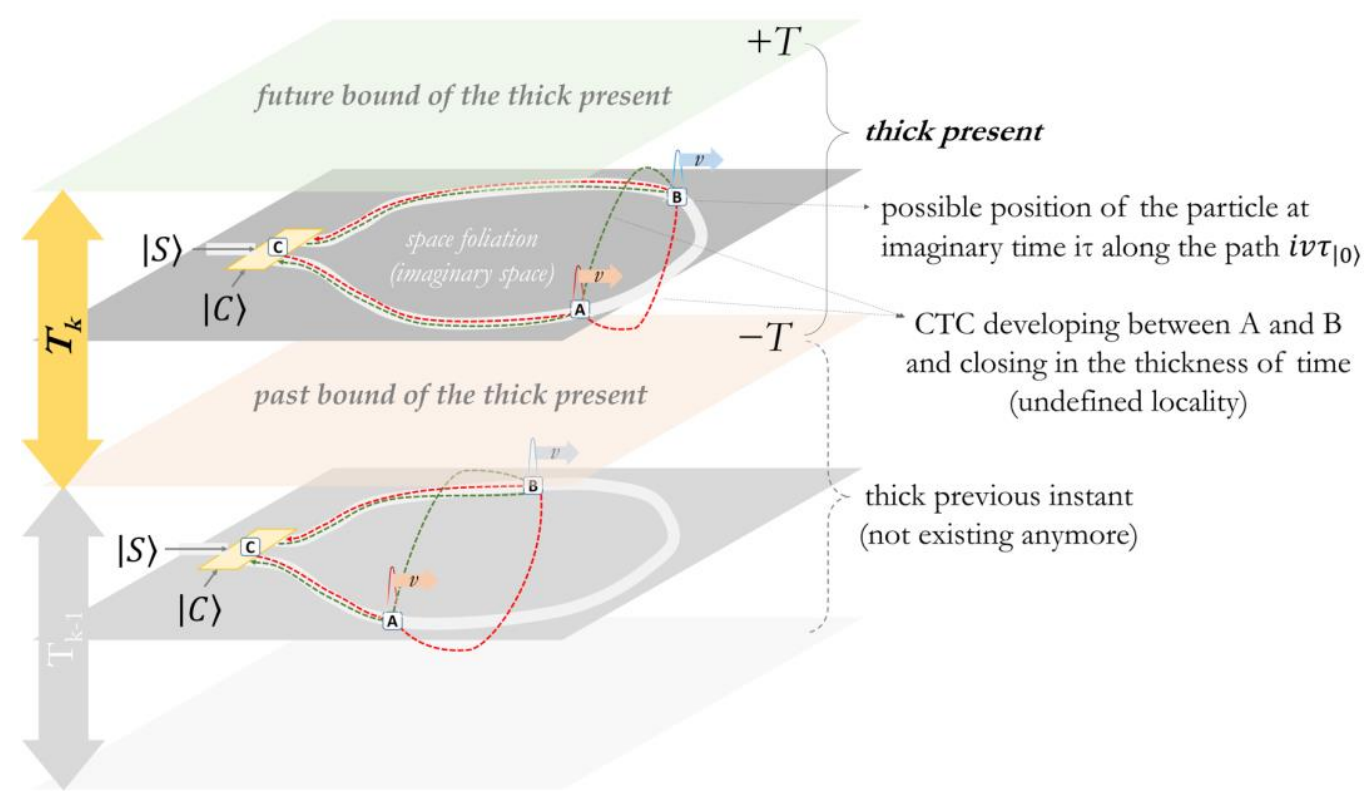

Figure 4. The imaginary space emerges from the information potential in the thick present and it is described through an imaginary time. CTC in the thickness are expression of non-local correlations.

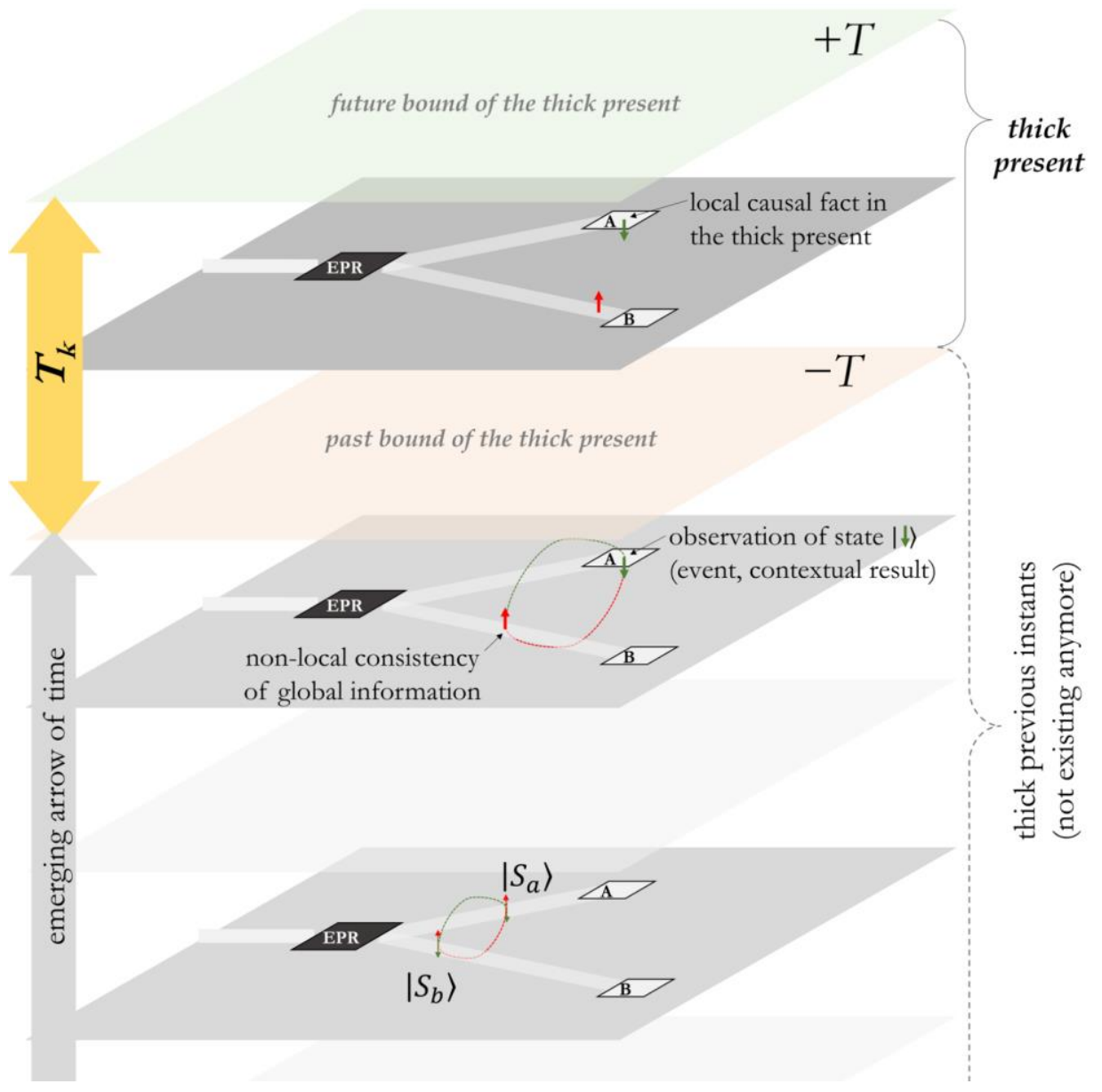

Figure 5. Successive snapshots of the imaginary space foliation, from the EPR pair generation to the measurement at Alice's location. The information potential persists along the successive instants through the entanglement/CTC as long as it is undetermined. When A defines a contextual outcome in her measurement, the state of the particle directed towards B is coherently defined so that the information keeps a global logical consistency within the thick present. 
The given description seems to pose on similar footing a spatial superposition of paths and an entanglement in time expressed as UCO, but this needs further clarification.

Chiribella et al. showed in Ref. [47] that the combination of entanglement-breaking channels in indefinite order could become a perfect quantum channel, while this is not true if the channels are in parallel (spatial superposition), highlighting a possible fundamental difference between space and time.

To understand the results reported in Ref. [47] in the context of a thick present, we should note that a channel from $S$ to $R$ is entanglement-breaking "along the path from $S$ to $R$ ". When the channels are in series but with "undefined order" thanks to a controller qubit, we are implicitly assuming that the coherence with the controller has persisted along the time of traversal of both channels (as well as in the connections to "go back to $S$ " before entering the next channel), defining coherent imaginary paths, still persisting at $R$ before any measurement on the controller. The resulting CTC in the imaginary time (coherent along the thick present instants even after the transmission over both channels) defines the UCO through the controller qubit and represents the additional quantum resource where the information persists identical along the communication (as for the channel $C_{+}$in [47]). When the channels are in parallel, after the time of traversal there are no CTC at $C$ or $R$ that could be used as an available quantum resource to encode the transmitted information: the entanglement on the "which way" would only define a causal branch, eventually selecting a single channel which would still be entanglement breaking.

\section{Towards a holographic perspective}

We have proposed a description of spacetime as a spacelike foliation emerging from a time symmetric thick present information potential $\boldsymbol{T}_{\boldsymbol{k}}$ along an imaginary time of motion. This potential represents the information of entanglement, manifested as undefined causality and non-locality and encoded in the thick present as CTC or "memory loops".

Even if not always in a Presentism perspective, several research groups are actively investigating the relations between entanglement, information and QG.

Holographic theories (introduced by Susskind in [48]) describes spacetime as emerging from the entanglement among distant quanta of space, encoded in a bulk region of which spacetime is the boundary. Extending these concepts in a Presentism context, the potential of the present $T_{k}$ would be equivalent (in a time symmetric description) to a pair of symmetric bulk regions, from $(2 k-1) T$ to $2 k T$ and from $(2 k+1) T$ to $2 k T$ respectively, of which the space foliation is the common boundary at $2 k T$.

Following the insights of a spacetime emerging from the information of entanglement among the quanta of space, if particles as well could be described as emerging from information, we can consider non-locality in the emerging foliation as the chances of being in multiple points in the imaginary space "at once", as if there were an imaginary ER bridge entangling the distant quanta of space.

In this sense, quantum tunneling in a spacelike foliation should be described as an entanglement in space and a connection through the real fourth dimension of spacetime: the thickness of the current instant in which everything could be interconnected.

Being time symmetric, this connection and non-local potential persists in the present as the superposition of a forward and a backward wave from $(2 k-1) T$ and $(2 k+1) T$ respectively, in a resulting CTC which intersects the foliation in the distant entangled quanta of space.

In the proposed perspective, we could eventually consider the non-local information represented in the wave function of an elementary particle as the superposition of a probabilistic "bundle of CTC" connecting different locations in the imaginary space.

This probabilistic ensemble of memory loops encodes the local information potential and embroiders the otherwise flat fabric emerging along the imaginary ict.

In a spacetime curved by the information of entanglement among the quanta of space, particles could then be intended as a "volume entangled on a common mode", locally persisting in each evolution cycle as a spacetime potential phase in respect to the global reference of action in the thick present. 
The proposed Presentism interpretation in a holographic perspective is introduced as a simple conjecture. Additional research is needed for a proper mathematical description of $\boldsymbol{T}_{k}$ in terms of entanglement among quanta of space, towards a full comprehension of its encoding in the symmetric bulks and of its relation with the gravitational potential.

Figure 6 graphically illustrates the holographic description of the thick present proposed, leaving further investigation on this path to a dedicated contribution.

Concluding, extending the famous equation of holographic theories, to represent the possible connection between time (existing as a real thick present potential $T_{k}$ ), space (emerging in each present instant along an imaginary time) and entanglement (as causally undefined or spatially non-local information of correlation among distant quanta of space) derived from the consistent superposition of outcomes of an open choice, we could maybe dare to conjecture, as limited Flatlanders living on a boundary, the following synthesis:

$$
E R=E P R=C T C @ T_{k}
$$

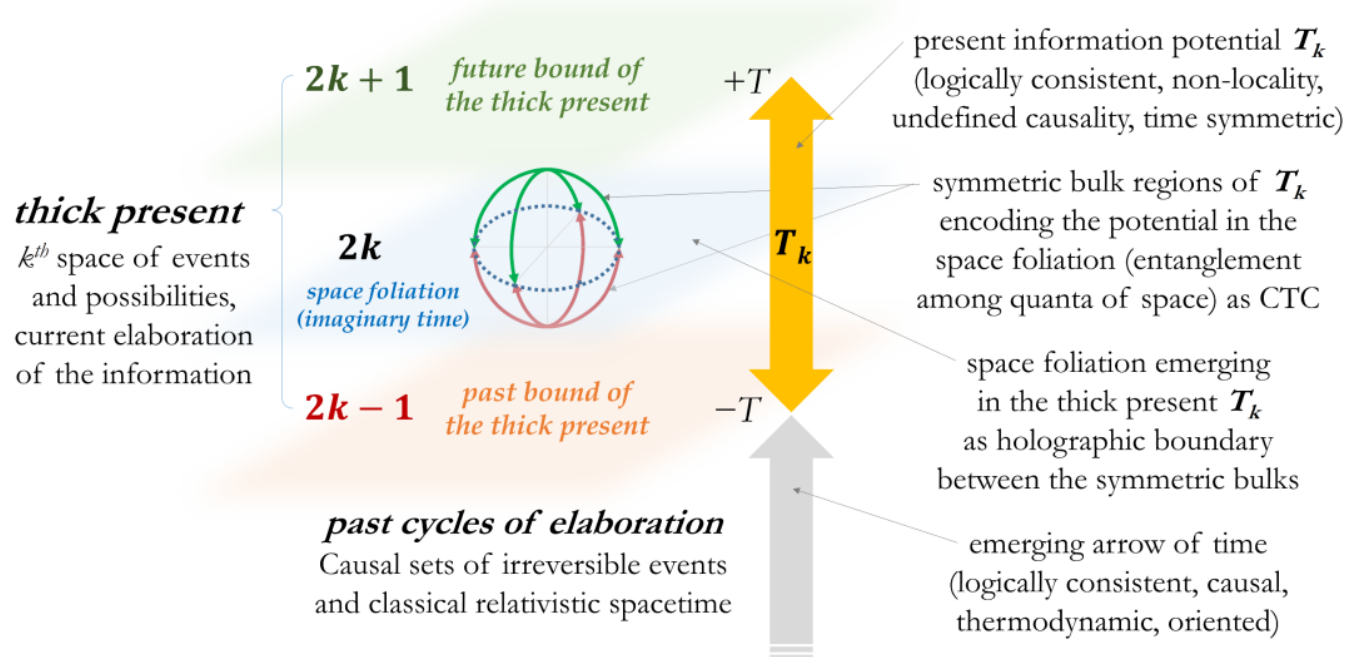

Figure 6. Graphics illustrating the space foliation at $2 k T$ in the thick present as the boundary between 2 symmetric bulk regions extending from $(2 k-1) T$ to $2 k T$ and from $(2 k+1) T$ to $2 k T$. The bulks encode the information $\boldsymbol{T}_{k}$ (intended in a holographic perspective as entanglement among quanta of space) from which spacetime emerges. CTC in the thickness of the present represent non-local potential, while are equivalent to undefined orders of traversed points when developing in the imaginary time.

\section{Synthesis and outlook}

In this paper we have discussed an interpretation on the nature of time and a proposal on the relation of entanglement with information, undefined causality and non-locality. We have investigated several descriptions of time, connecting elements from the different perspectives in the search for a common intersection, and eventually concluded the existence of a thick present as the only element of reality in an emerging axis of time.

A thick present exists between a causal past of irreversible events and an open future. Within a thick present, we have considered a global information potential $T_{k}$ in a time symmetric description, from $(2 k-1) T$ to $(2 k+1) T$. The potential $\boldsymbol{T}_{\boldsymbol{k}}$ has been pictured as a logically consistent information evolving along the thick present instants, coherent with what happened and what could happen. In each instant, spacetime has been considered as a spacelike foliation emerging from the information in the thick present.

Following a QIS perspective, we have connected the thick present temporal extension to a spacetime "sampling rate" and a discrete elaboration of the information potential, towards the realization of a discrete time, connected to absolute references (such as the Planck units) and not in contrast with a relativistic description of the physics of time as experienced and measured by local observers. 
The atomic elaboration of the information of $\boldsymbol{T}_{k}$ has been proposed as an elementary quantum of action and the "fastest event" to evaluate differences, acting as a reference to consistently compare any relativistic perspective on spacetime among observers.

We have concluded the first part of the paper clarifying how a Presentism perspective could reconcile philosophy, neuroscience and physics, and which could be the open challenges towards a quantum description of spacetime based on a thick present potential.

In the second part of this contribution, we have investigated indefinite causal orders, to understand how their information potential could be described and persist along an evolution occurring in thick present instants.

Thanks to a parallel with a C-NOT quantum gateway, we have interpreted entanglement as the coherent superposition of the possible outcomes of an "open choice", in a logically consistent potential that discards under/over-determined solutions.

Following a paths integral approach on the circuit implementing the undefined orders, we have described the evolution of the system as superposed imaginary paths developing in the thick present along an imaginary time of motion.

Considering a time symmetric description of the potential in the thick present, we have interpreted the entanglement in time order as the superposition of a forward and backward wave evolving in the imaginary time of motion along the circuit, closing on the controller qubit in an imaginary closed path and eventually a CTC.

CTC are introduced to represent entanglement in both time and space, manifested in the emerging spacetime as the information of undefined causality and non-locality.

In a QIS description, CTC in the thick present have been considered equivalent to "memory-loops" able to encode the information of an open choice of which the outcome is logically consistent and undetermined at the most fundamental level (until observed and contextually defined). When developing along the imaginary time of motion in the space foliation, these CTC represent indefinite orders (of imaginary quanta of space traversed) and an undefined causality, while encode non-local correlations in the imaginary space (spatial entanglement) if their path develops through the thickness of the present instant.

In the final part of the contribution, we have investigated a possible interpretation of the thick present in a holographic perspective.

In the context of a spacetime emerging as flat along an imaginary ict and curved by the logically consistent information of entanglement among quanta of space, the description of the potential through CTC in a time-symmetric thick present $T_{k}$ has been conceptually extended to the information expressed in the wave function of an elementary particle.

Massive particles have been interpreted as the potential persisting in a probabilistic bundle of CTC, that embroiders and deforms the fabric of spacetime, entangling in each present evolution cycle the imaginary quanta of the emerging space foliation.

Following the ideas proposed in the contribution, we have concluded conjecturing a possible extension of the famous equation of holographic theories to ER $=E P R=C T C @ T_{k}$ and left additional research on this path to a dedicated contribution.

The descriptions of time and of entanglement given in this paper are missing explicit mathematical derivations and could be seen as just conjectures inspired by logic and QIS. Nevertheless, the concept of a time symmetric thick present potential $\boldsymbol{T}_{k}$ that encodes through CTC the information of entanglement (as undefined causality and non-locality in the emerging space foliation), seems a promising starting framework for the interpretation of our Universe in terms of information.

The hope is that future works on this path may offer additional insights into the possible ontological nature of information in the emergence of spacetime, towards a proper quantum description of gravity and a more profound understanding of our Universe.

Funding: This research received no external funding.

Conflicts of Interest: The author declares no conflict of interest. 


\section{References}

[1] D. Buonomano and C. Rovelli, "Bridging the neuroscience and physics of time," in Time and Science, Lestienne \& Harris, 2021.

[2] J. Conway and S. Kochen, "The Free Will Theorem," Foundations of Physics, vol. 36, p. 1441-1473, 2006.

[3] F. Dyson, "Thought Experiments in Honor of John Archibald Wheeler," Science and Ultimate Reality, Cambridge University Press, p. 89, 2004.

[4] L. Smolin, "The dynamics of difference," Found Phys, vol. 48, 2018.

[5] T. C. N. Boekholt, S. F. Portegies Zwart and M. Valtonen, "Gargantuan chaotic gravitational three-body systems and their irreversibility to the Planck length," Monthly Notices of the Royal Astronomical Society, vol. 493, no. 3, 2020.

[6] S. Watanabe, "Symmetry of physical laws. Part III. Prediction and retrodiction.," Reviews of Modern Physics, vol. 27, p. 179-186, 1955.

[7] V. Scarani and F. Buscemi, "Fluctuation theorems from Bayesian retrodiction," Phys. Rev. E, vol. 103, no. 052111, 2021.

[8] V. Scarani, F. Buscemi and C. C. Aw, "Fluctuation Theorems with Retrodiction rather than Reverse Processes," AVS Quantum Sci., vol. 3, pp. 045601 doi.org/10.1116/5.0060893, 2021.

[9] Ä. Baumeler, "Causal Loops: Logically Consistent Correlations, Time Travel, and Computation," PhD Thesis, Università della Svizzera Italiana, 2017.

[10] E. Cohen, M. Cortes, A. C. Elitzur and L. Smolin, "Realism and causality I: Pilot wave and retrocausal models as possible facilitators," Phys. Rev. D, vol. 102, no. 12, 2020.

[11] E. Cohen, M. Cortes, A. C. Elitzur and L. Smolin, "Realism and causality II: Retrocausality in energetic causal sets," Phys. Rev. D, vol. 102, no. 12, 2020.

[12] J. Tallant and D. Ingram, "The rotten core of presentism," Synthese, vol. 199, pp. 39693991 - doi.org/10.1007/s11229-020-02965-w, 2021.

[13] C. Mariani and G. Torrengo, "The Indeterminate Present and the Open Future," Synthese, vol. 199, pp. 3923-3944 - doi.org/10.1007/s11229-020-02963-y, 2021.

[14] N. Gisin, "Classical and intuitionistic mathematical languages shape our understanding of time in physics," Nature Physics, vol. 16, pp. 114-116, 2020.

[15] L. Smolin and C. Verde, "The quantum mechanics of the present," arXiv:2104.09945v1, 2021.

[16] A. Schlatter, "On the Principle of Synchronization," Entropy, vol. 20, pp. 741 doi:10.3390/e20100741, 2018.

[17] A. Schlatter, "On the Reality of Quantum Collapse and the Emergence of SpaceTime," Entropy, no. 21, pp. 323 - doi:10.3390/e21030323, 2019.

[18] A. Schlatter, "On the Foundation of Space and Time by Quantum-Events," Found Phys, vol. 52, pp. 7 - doi.org/10.1007/s10701-021-00526-w, 2022.

[19] S. C. Fletcher, "Light Clocks and the Clock hypothesis," Found Phys, vol. 43, pp. 13691383 - DOI 10.1007/s10701-013-9751-3, 2013.

[20] N. Margolus, "Finite-State Classical Mechanics," RC, 2018.

[21] G. Jaroszkiewicz, Quantized Detector Networks: The Theory of Observation, Cambridge University Press, 2017.

[22] L. Hardy, "Foliable Operational Structures for General Probabilistic Theories," in Deep Beauty Understanding the Quantum World through Mathematical Innovation, Cambridge University Press, 2011, pp. 409-442 - doi.org/10.1017/CBO9780511976971.013.

[23] G. M. D'Ariano, "Physics as quantum information processing," in AIP Conference Proceedings, 2011. 
[24] G. Chiribella, G. M. D'Ariano and P. Perinotti, "Informational derivation of quantum theory," PHYSICAL REVIEW A, vol. 84, no. 012311, 2011.

[25] L. Hardy, "Time Symmetry in Operational Theories," arXiv:2104.00071v1, 2021.

[26] O. Oreshkov and N. Cerf, "Operational formulation of time reversal in quantum theory," Nature Phys, vol. 11, pp. 853-858 - doi.org/10.1038/nphys3414, 2015.

[27] A. Di Biagio, P. Donà and C. Rovelli, "The arrow of time in operational formulations of quantum theory," Quantum, vol. 5, pp. 520 - doi.org/10.22331/q-2021-08-09-520, 2021.

[28] J. Christian, "Passage of Time in a Planck Scale Rooted Local Inertial Structure," International Journal of Modern Physics D, vol. 13, no. 06, pp. 1037-1071, 2004.

[29] C. Rovelli, "The layers that build up the notion of time," arXiv:2105.00540, 2021.

[30] M. Zych, F. Costa, I. Pikovski and C. Brukner, "Bell's theorem for temporal order," Nature Communication, vol. 10, no. 3772, 2019.

[31] G. Rubino, L. A. Rozema, F. Massa, M. Araújo, M. Zych, Č. Brukner and P. Walther, "Experimental Entanglement of Temporal Orders," Quantum Information and Measurement, vol. Quantum Technologies, p. paper S3B.3, 2019.

[32] G. Rubino, L. A. Rozema, A. Felix, M. Araújo, J. M. Zeuner, L. M. Procopio, Č. Brukner and P. Walther, "Experimental verification of an indefinite causal order," Science Advance, vol. 3, p. e1602589, 2017.

[33] L. M. Procopio, A. Moqanaki, M. Araújo, F. Costa, A. Calafell, E. G. Dowd, D. R. Hamel, L. A. Rozema, Č. Brukner and P. Walther, "Experimental superposition of orders of quantum gates," Nature Communication, vol. 6, p. 7913, 2015.

[34] G. Chiribella and Z. Liu, "Quantum operations with indefinite time direction," arXiv:2012.03859, 2021.

[35] S. W. Hawking, The Universe in a Nutshell, Bantam Spectra, 2001.

[36] N. L. Diaz, J. M. Matera and R. Rossignoli, "Spacetime Quantum Actions," Phys. Rev. D, vol. 103, pp. 065011 - DOI: 10.1103/PhysRevD.103.065011, 2021.

[37] B. Koch and E. Muñoz, "Path integral of the relativistic point particle in Minkowski space," Phys. Rev. D, vol. 103, pp. 105025 - DOI: 10.1103/PhysRevD.103.105025, 2021.

[38] K. Gödel, "An example of a new type of cosmological solutions of Einstein's field equations of gravitation," Reviews of Modern Physics, vol. 21, pp. 447-450, 1949.

[39] D. Lewis, "The paradoxes of time travel," American Philosophical Quarterly, vol. April, p. 145.152, 1976.

[40] H. Nikolic, "Causal paradoxes: a conflict between relativity and the arrow of time," Foundation of Physics Letters, vol. 19, pp. 259-257, 2006.

[41] C. Rovelli, "Can we travel to the past? Irreversible physics along closed timelike curves," arXiv:1912.04702v2, 2019.

[42] C. F. Paganini, "No events on closed causal curves," arXiv:2005.05748v4, 2021.

[43] J. Barrett, R. Lorenz and O. Oreshkov, "Cyclic quantum causal models," Nat Commun, vol. 12, pp. 885 - doi.org/10.1038/s41467-020-20456-x, 2021.

[44] A. Baumeler, A. S. Gilani and J. Rashid, "Unlimited non-causal correlations and their relation to non-locality," arXiv:2104.06234v1, 2021.

[45] J. S. Bell, "On the Einstein Podolsky Rosen paradox," Physics, vol. 1, p. 195-200, 1964.

[46] S. Kochen and E. P. Specker, "The Problem of Hidden Variables in Quantum Mechanics," Springer Netherlands, p. 293-328, 1975.

[47] G. Chiribella, "Indefinite causal order enables perfect quantum communication with zero capacity channels," New J. Phys., vol. 23, no. 033039, 2021.

[48] L. Susskind, "The World as a Hologram," J. Math. Phys, vol. 36, no. 11, pp. 6377-6396 - doi:10.1063/1.531249, 1995. 